\title{
Analysis of the oxidative stress inhibition potentials of Artemisia annua and its bioactive compounds through in vitro and in silico studies
}

\author{
Stephen Adakole EJEMBI ${ }^{1 *}$, Titilayo Omolara JOHNSON ${ }^{1}$, Jonathan Dingkwoet \\ DABAK $^{1}$, Augustina Oduje AKINSANMI ${ }^{1}$, Jane-Rose Ifuanyachi $\mathrm{OCHE}^{1}$ and Timothy \\ FRANCIS $^{2}$
}

${ }^{I}$ Department of Biochemistry, Faculty of Basic Medical Sciences; $\quad{ }^{2}$ Department of Pharmacology, Faculty of Pharmaceutical Sciences; University of Jos, P.M.B. 2084, Jos. Plateau State, Nigeria.

Received 21 $1^{\text {st }}$ June 2021; Accepted $3^{\text {rd }}$ August 2021

\begin{abstract}
Oxidative stress overwhelms the antioxidant mechanisms of living systems, with active involvement in the pathogenesis of several diseases. Natives of Gangnim in the Plateau State of Nigeria may be unknowingly endowed with some protective advantages against oxidative stress for their habitual consumption of Artemisia annua tea. The antioxidant activities of $A$. аппиа extracts were determined using in vitro methods and the inhibitory potentials of twenty-nine (29) bioactive compounds of the plant against oxidative stress target proteins were assessed through molecular docking analysis. These extracts showed significantly high activities in scavenging nitric oxide, 2,2diphenyl-1-picrylhydrazyl (DPPH) and reducing ferric $\left(\mathrm{Fe}^{3+}\right)$ to ferrous $\left(\mathrm{Fe}^{2+}\right)$ iron. Virtually, none of the bioactive compounds binds to the active site of the antioxidant protein targets. Rather, $72.41,93.10$ and $75.86 \%$ of these compounds bind with high binding affinity to the activator binding sites of superoxide dismutase (SOD), glutathione peroxidase $(G S H-P x)$ and catalase $(C A T)$ respectively. 7,8 -dimethylalloxazine $(-8.10 \mathrm{kcal} / \mathrm{mol})$ ranked highest as a prospective inhibitor of xanthine oxidase $(X O X)$. The antioxidant activity exhibited by the extracts of the locally cultivated A. аппи a and the molecular interactions of its bioactive compounds against the protein targets used predict that oxidative stress inhibition could be effectively achieved with these phytochemicals.
\end{abstract}

Keywords: Artemisia аппиа, antioxidant, antioxidant protein targets, molecular docking, oxidative stress

\section{INTRODUCTION}

The consumption of locally brewed 'tea' and decoction of $A$. апnиa has been a common practice among the natives of Gangnim, in Langtang South Local Government Area (L.G.A.) of Plateau State, (North-Central) Nigeria, with the hope of treating malaria. Interestingly, past and recent studies had shown that the Gangnim people are not on the wrong [1-2]. For more than two millennia, A. аппиа has been in the spotlight as a medicinal plant in Chinese Pharmacopoeia for the cure of malaria [3-5]. Malaria cure efficacy of this medicinal plant has been a trending issue in several studies. There are possibilities, however, that the consumption of A. апnиа confers more health benefits than just malaria cure.

*Correspondence. E-mail: stevcitifee44@gmail.com Tel: +234-8030474883.

ISSN 0189-8442

2021. Published by Faculty of Pharmaceutical Sciences, University of Jos, Nigeria. Under Creative Commons Attribution-Non-Commercial 4.0 International License. https://creativecommons.org/licenses/by-nc/4.0/ 
One of such possibilities could be the potential to prevent or ameliorate the deleterious effect of oxidative stress. This study was carried out, assessing this possibility by the evaluation of the antioxidant activity and oxidative stress inhibition potential of extracts from leaf and seed, as well as some bioactive compounds of the plant. Oxidative stress was coined to mean an oxidantantioxidant imbalance that results in an overwhelming activity of free radicals against the antioxidant system of living cells [6-7]. The involvement of free radicals (radical generating agents) in a number of chronic diseases [8-9] cannot be overemphasized. The mitochondrial respiratory chain, NAD $(\mathrm{P}) \mathrm{H}$ oxidase $(N O X)$, xanthine oxidase $(X O X)$ and nitric oxide synthases $(N O S)$ had been reported as the main sources of endogenous free radicals in blood vessels [6]. In short, every system that involves the use of enzymes and oxygen to perform any function in living cells is exposed to free radical reactions. When in excess, they have the potential to cause oxidative damage to DNA, proteins, lipids and other small cellular molecules $[10,11]$ by 'stealing' electrons from these molecules [9]. This electron 'theft' by free radicals may eventually cause diabetes, cancer, and several degenerative diseases in humans [12]. Inagi [6(p139)] opined that "host cells are endowed with a number of antioxidant systems to limit (free radical) levels. $\mathrm{O}_{2}^{-}$may be dismutated by a family of SODs to (a less reactive species) $\mathrm{H}_{2} \mathrm{O}_{2} \cdot \mathrm{H}_{2} \mathrm{O}_{2}$ can be scavenged to water by $C A T$ or by glutathione peroxidase (GPx) in the presence of GSH'. Studies have shown that antioxidant supplements are in use to boost the activity of the built-in antioxidant system in order to combat oxidative stress $[9,13]$. Unfortunately, some of these synthetic antioxidants are reported to be involved in several diseases and their use had been discontinued in many developed countries [14], promoting the need for health professionals to search for alternative sources of antioxidants based on natural origin, which may be safer, more effective and economical, preferably from plant materials based on indigenous resources [15].

Historically, plants are well known to contain a wide variety of free radical scavenging molecules, such as flavonoids, carotenoids, and vitamins [16]. Convincingly, the curative properties of medicinal plants are conceivably due to the presence of these secondary metabolites and Artemisia annua, commonly known as "sweet wormwood" in English, "qinghao" in Chinese and "armoise annuelle" in French [3] is among the potential medicinal plants of antioxidant properties, exhibiting several promising characteristics of which over five hundred $(>500)$ bioactive compounds had been reported to have been characterized [17] including the popular antimalarial drug, artemisinin [5].

The results of our study beep with optimism that oxidative stress chemoprotection with phytochemicals from A. annua plant could probably be one of the most feasible approaches for the cure of diseases for which their pathogenesis and progression are traceable to oxidative stress.

\section{EXPERIMENTAL METHODS}

Plant sample collection. The leaf and seed of A. annua used in this study were harvested near flowering stage from the nursery farm of the Centre for Biotechnology and Genetic Engineering sited in Gangnim, Langtang South L.G.A. of Plateau State, Nigeria. The freshly harvested plant was identified and authenticated by Mr. J.J. Azila of Federal School of Forestry, University of Jos, Jos, Plateau State and assigned a voucher number: No. FHJ 249 of herbarium specimen.

Aqueous extraction. The method described by Asuzu [18] was used for the aqueous extraction. The filtrate was concentrated using a hot water bath at $50^{\circ} \mathrm{C}$ and air-dried. Dried extracts were labeled $A A L$ (aqueous leaf extract of $A$. annua) and $A A S$ (aqueous seed 
extract of $A$. апnua) and kept in the freezer until it was ready to be used.

Methanol extraction. Methanol extraction was carried out, using the method described by Chu, et al. [19]. The filtrate was poured on a flat plastic tray and left to air dry. Dried extracts were labeled $M A L$ (methanol leaf extract of $A$. annua) and $M A S$ (methanol seed extract of $A$. аnnua) and stored in the freezer until it was ready to be used.

Qualitative and quantitative phytochemical determination. Qualitative phytochemical analysis was carried out using standard methods [20-22]. The total phenolic and flavonoid contents of extracts were determined using the method described by Singleton, et al. [23] and Meda, et al. [24] respectively using UV/Vis spectrophotometry.

In vitro antioxidant analysis of extracts of $\boldsymbol{A}$. annua leaf and seed. The method by Oyaizu [25] was followed to evaluate the ironreducing property, 2,2-diphenyl-1picrylhydrazyl (DPPH) radical scavenging assay according to Gyamfi, et al. [26] and nitric oxide radical scavenging activity according to Marcocci, et al. [27].

\section{Molecular docking}

Crystal structure of target proteins and ligands collection. Twenty-nine (29) previously identified and characterized bioactive compounds of $A$. annua in a review by Nigam, et al. [5] and standard ligands were docked against eight selected protein targets (Table 1) alongside known inhibitors of these targets. $S O D, G S H-P x$ and $C A T$ were used for the in silico evaluation of antioxidant activity while Xanthine oxidase $(X O X)$, NADPH oxidase (NOX), caspase-1 (Casp-1), endothelial nitric oxide synthase (eNOS) and induced nitric oxide synthase (iNOS) were the other five targets selected as a result of their direct involvement in the generation of free radicals. Crystal structures of these targets were accessed from the protein data bank [28] with their PDB IDs (Table 1). Structure data file (SDF) format of bioactive compounds of $A$. апnиa, standard ligands reported to be agonists of antioxidant targets (trehalose, metformin and vitamin E), and inhibitors of oxidative stress proteins (asymmetric dimethylarginine (ADMA), indoprofen, 6-mercaptopurine, and VAS2870) were obtained from PubChem database. The PubChem CIDs of ligands are shown in Tables $4 \& 5$.

Proteins and ligands preparation, docking and Post docking analysis. Prior to the docking analysis, all non-standard residues (all bound ligands, cofactors, and water molecules) were removed from our target proteins. The Proteins were checked for polar hydrogen, and torsion bonds of the ligands were selected and defined. Gasteiger charges were computed, and the AutoDock atom types were defined using AutoDock version 4.2 as described by Singh, et al. [29]. AutoDock vina in PyRx software was used to perform the molecular docking while the post docking analysis was carried out using Chimera 1.14 at 100 steepest minimizations and 10 steps of the conjugate gradient. A relax constraints of 0.4 Angstrom and $20^{\circ}$ were used to determine the conventional $\mathrm{H}^{+}$bond interaction of ligands with the side chains of the amino acids of targets. Chimera 1.14 and Discovery Studio 2020 were used for the 3D (3-dimensional) and 2D (2-dimensional) interactions, respectively, adopting the method described by Rana, et al. [30] and Johnson, et al. [31].

Data analysis. The results in triplicates of the in vitro assays were collated and expressed as mean \pm standard error mean (SEM). Analysis of Variance (ANOVA) (followed by the Bonferioni post-hoc t-test) was used for result analysis and significance difference at $P<0.05$, using Microsoft Excel 2010. The binding affinity $(-\Delta \mathrm{G} \mathrm{kcal} / \mathrm{mol})$ of ligands were recorded as generated by the computational tools unaltered. 


\section{RESULTS AND DISCUSSION}

Phyto constituents of extracts of $A$. annua leaf and seed. The brewing of $A$. аnnua plant for tea by Gangnim community dwellers is justifiable: the results of our study (Table 2) reveal that flavonoids and phenols were higher in the leaf aqueous extract than the methanol extraction. Emmanuel, et al. [1] reported the presence of similar classes of phytochemicals in their n-hexane extracts of $A$. аппи a from the same community. The phytochemicals of $A$. аппиа are predominantly terpenoids (in particular sesquiterpene lactones), flavonoids, coumarins and other shikimate metabolites (Table 3), of which A. аnnua is currently the only commercial source of the sesquiterpene lactone, Artemisinin [17,32,43]. Studies have shown that the majority of these groups of phytochemicals have been established to have significant antioxidant activity [33] and hot water is an effective extraction solvent [3,34]. Phenolics, according to Oboh and Ademosun [35] are capable of scavenging free radicals, chelating metals, activating antioxidant enzymes, reducing $\alpha$-tocopherol radicals, and inhibiting oxidases.

In vitro antioxidant activity of extracts of $A$. annua leaf and seed. Results (Figure 1) reveal a significant difference in the iron-reducing activity of $M A S$ at $27.28 \mu \mathrm{g} / \mathrm{ml}$, with $\mathrm{p}<0.05$ measured in ascorbic acid equivalent (AAE). The reduction of ferric iron $\left(\mathrm{Fe}^{3+}\right)$ to ferrous iron $\left(\mathrm{Fe}^{2+}\right)$ and the chelation of excess iron is very crucial in the antioxidant mechanism $[32,33,36]$. The extracts displayed significantly high iron-reducing activity with $M A L$ showing the highest activity $(0.066 \mu \mathrm{g}$ AAE/mg extracts). Phenolic compounds are known to protect against a wide range of diseases including certain types of cancers and part of the antioxidant effects of flavonoids is in their ability to chelate metals such as iron and copper [32].

The results of the DPPH scavenging assay (Figure 2) show a significant difference in the activity of extracts at $83.3 \mu \mathrm{g} / \mathrm{ml}$. The results of nitric oxide scavenging activity (Figure 3) revealed that all the extracts followed a normal curve pattern and their activity is dose-dependent. Reactive peroxynitrite $\left(\mathrm{ONOO}^{-}\right)$, a product from the reaction of nitric oxide (NO) and superoxide anion, is known to produce serious toxic reactions with protein, lipids and nucleic acids. The scavenging of NO by extracts of $A$. апnиa may prevent the pathological effect caused by excessive generation of ONOO-[37].

Binding energies of bioactive compounds of A. annua plants. Binding affinity (- $\Delta \mathrm{G}$ $\mathrm{kcal} / \mathrm{mol}$ ) of ligands which had been identified as activators of SOD, CAT and GSH-Px, and those of standard protein target inhibitors are displayed in Table 4 and Table 5 respectively. $S O D, C A T$ and GSH-Px (targets) are involved in antioxidant pathways as described in biochemistry [38] form an excellent choice for this study. The study of Rana, et al. reported that some phytochemicals "act as agonists of (antioxidant enzymes) and increase the activity of SOD, GSH-PX and CAT' [30(p4)].

The results of this study show that 3,5di-O-caffeoylquinic acid, 5-O-[(E)caffeoyl]quinic acid, Daucosterol, 7,8dimethylalloxazine, Methyl-3,4-di-Ocaffeoylquinic acid and Methyl-3,5-di-Ocaffeoylquinic acid ranked highest with docking score ranging from -7.30 to -6.50 $(\mathrm{kcal} / \mathrm{mol})$ compared to $-5.50(\mathrm{kcal} / \mathrm{mol})$ of Trehalose (standard ligand) against SOD. The docking scores of 3,4-di-O-caffeoylquinic acid, 3,5-di-O-caffeoylquinic acid, 4,5-di-Ocaffeoylquinic acid, Artemisinin, $\beta$-sitosterol, 1,3-di-O-caffeoylquinic acid and Daucosterol were higher than that of standard ligand (Metformin $(-5.40 \mathrm{kcal} / \mathrm{mol})$ ) with 4,5-di-Ocaffeoylquinic being the highest $(-9.40$ $\mathrm{kcal} / \mathrm{mol}$ ) and closely followed by $\beta$-sitosterol $(-9.30 \mathrm{kcal} / \mathrm{mol})$ against $C A T$. Similarly, 3,5di-O-caffeoylquinic acid, $\beta$-sitosterol, 1,3-diO-caffeoylquinic acid, Daucosterol and Methyl-3,5-di-O-caffeoylquinic acid ranked highest in their binding affinity to $G S H-P x$ 
compared to Vitamin E $(-5.80 \mathrm{kcal} / \mathrm{mol})$. Overall, $72.41,93.10$ and $75.86 \%$ of these bioactive compounds are likely to be agonists of SOD, CAT and GSH-Px respectively for their high and promising binding energies towards the activator sites of these proteins. Virtually, none of the bioactive compounds of A. апnиa in this study had significant binding affinity for the active sites of these antioxidant proteins.

Concurrently, 3,4-di-O-caffeoylquinic acid, 3,5-di-O-caffeoylquinic acid, 4,5-di-Ocaffeoylquinic acid, $\beta$-sitosterol, Daucosterol and 7,8-dimethylalloxazine were ligands with topmost binding affinities for iNOS, eNOS, Casp-1, XOX and NOX compared to standard inhibitors of each protein (Table 5). Only in the docking against NOX is VAS2870 (-7.50 $\mathrm{kcal} / \mathrm{mol}$ ) higher than 4,5-di-O-caffeoylquinic acid (-7.30 kcal/mol). Generally, 93.10, $100.00,13.79,96.55$ and $17.24 \%$ of these bioactive compounds of $A$. annua have high prospects of being $i N O S$, eNOS, Casp-1, XOX and NOX inhibitors, respectively.

Nitric oxide is produced in living cells via nitric oxide synthases (NOS). Studies have shown that three isoforms of NOS: eNOS (endothelial NOS), nNOS (neuronal NOS) and iNOS (inducible NOS), had been identified [39]. Our study made use of two of these isoforms with emphasis on the iNOS. "Inducible nitric oxide synthase is a key enzyme responsible for the production of nitric oxide (NO) and it plays an important role in oxidative stress" [37(p398)].

Table 1: Target proteins and their binding site grid-box dimension

\begin{tabular}{|c|c|c|c|c|c|c|c|}
\hline & \multicolumn{3}{|c|}{ Protein targets } & \multirow{2}{*}{$\begin{array}{c}\text { PDB } \\
\text { ID }\end{array}$} & \multicolumn{3}{|c|}{ Dimension } \\
\hline & $\#$ & Name & Abbrev & & $\mathrm{x}$ & $\mathrm{Y}$ & $\mathrm{Z}$ \\
\hline \multirow{8}{*}{ Blind docking } & 1 & Superoxide dismutase & $S O D$ & 1PU0 & 33.5431 & 44.5211 & 41.6160 \\
\hline & 2 & Catalase & $C A T$ & 1QQW & 68.1248 & 80.4353 & 75.6187 \\
\hline & 3 & Glutathione peroxidase & $G S H-P x$ & $2 \mathrm{I} 3 \mathrm{Y}$ & 42.7357 & 48.5262 & 50.4933 \\
\hline & 4 & Xanthine oxidase & $X O X$ & 3B9J & 36.9225 & 46.5775 & 52.5419 \\
\hline & 5 & NADPH oxidase & $N O X$ & $1 \mathrm{OEY}$ & 28.9431 & 45.4195 & 34.4152 \\
\hline & 6 & Caspase-1 & Casp-1 & 5MMV & 43.5554 & 51.3561 & 66.1002 \\
\hline & 7 & Endothelial nitric oxide synthase & $e N O S$ & $3 \mathrm{NOS}$ & 57.7616 & 72.4500 & 53.7818 \\
\hline & 8 & inducible nitric oxide synthase & $i N O S$ & 2NSI & 45.5762 & 74.8195 & 60.7555 \\
\hline Active site & 1 & Xanthine oxidase & $X O X$ & 3B9J & 14.3699 & 15.2060 & 15.7181 \\
\hline directed docking & 2 & Caspase-1 & Casp-1 & $5 \mathrm{MMV}$ & 6.2216 & 11.2864 & 7.5276 \\
\hline
\end{tabular}

Table 2: Results of qualitative and quantitative phytochemical analyses of extracts of $A$. annua leaf and seed

\begin{tabular}{|c|c|c|c|c|c|c|c|c|}
\hline \multirow{2}{*}{ Screened phytochemicals } & \multicolumn{2}{|r|}{$A A L$} & \multicolumn{2}{|r|}{$A A S$} & \multicolumn{2}{|r|}{$M A L$} & \multicolumn{2}{|r|}{$M A S$} \\
\hline & Qlt. & Quantitative & Qlt. & Quantitative & Qlt. & Quantitative & Qlt. & Quantitative \\
\hline Alkaloids & + & ND & + & ND & + & ND & - & ND \\
\hline $\begin{array}{c}\text { Flavonoids }(\mu \mathrm{gQUE} / \mathrm{mg} \\
\text { extract) }\end{array}$ & + & $0.033 \pm 0.002$ & + & $0.032 \pm 0.000$ & + & $0.029 \pm 0.000$ & + & $0.026 \pm 0.002^{\mathrm{a}}$ \\
\hline $\begin{array}{c}\text { Phenols }(\mu \mathrm{gGAE} / \mathrm{mg} \\
\text { extract })\end{array}$ & + & $0.119 \pm 0.010$ & + & $0.109 \pm 0.005$ & + & $0.104 \pm 0.009$ & + & $0.096 \pm 0.002$ \\
\hline Saponins & + & ND & + & ND & + & ND & + & ND \\
\hline Terpenes & + & ND & + & ND & - & ND & + & ND \\
\hline Cardiac Glycosides & + & ND & + & ND & - & ND & - & ND \\
\hline Balsam & + & ND & + & ND & + & ND & + & ND \\
\hline Carbohydrate & + & ND & + & ND & + & ND & + & ND \\
\hline Tannins & + & ND & + & ND & + & ND & + & ND \\
\hline Resins & + & $\mathrm{ND}$ & + & ND & + & $\mathrm{ND}$ & - & $\mathrm{ND}$ \\
\hline
\end{tabular}

+ Indicates the presence of the phytochemical; - indicates the absence of the phytochemical; ND-means Not determined; Values are presented in mean \pm standard error mean (SEM) of triplicate results $(\mathrm{n}=3)$. Superscript $\left(^{a}\right)$ indicates group that is statistically significant as compared to aqueous extract of $A$. annua seed $(A A S)$. Difference was considered significant at $\mathrm{p}<0.05$ Aqueous extract of $A$. annиa leaf $(A A L)$, aqueous extract of $A$. annua seed 
$(A A S)$, Methanol extract of A. апnи leaf $(M A L)$ and Methanol extract of A. annиa seed (MAS). QUE - quercetin equivalent; GAE - gallic acid equivalent; Qlt. = Qualitative.

Table 3: Bioactive compounds of A. аппиа and their class of phytochemicals

\begin{tabular}{|c|c|c|}
\hline Class of Phytochemicals & Sub-class & Bioactive compounds of A. аппиа \\
\hline \multirow{17}{*}{ 1. Polyphenols } & \multirow{8}{*}{ Phenolic acid (Chlorogenic acid) } & 3,4-di-O-caffeoylquinic acid \\
\hline & & 3,5-di-O-caffeoylquinic acid \\
\hline & & 4,5-di-O-caffeoylquinic acid \\
\hline & & 5-O-[(E)-caffeoyl] quinic acid \\
\hline & & 1,3-di-O-caffeoylquinic acid \\
\hline & & Methyl-3,4-di-O-caffeoylquinic acid \\
\hline & & Methyl-3,5-di-O-caffeoylquinic acid \\
\hline & & $\begin{array}{l}\text { p-nyaroxybenzorc acia } \\
\text { Salicylic acid }\end{array}$ \\
\hline & \multirow{3}{*}{ Cumarins } & Scopoletin \\
\hline & & Scoparone \\
\hline & & Scopolin \\
\hline & \multirow{5}{*}{ Flavonols } & Chrysosplenol D \\
\hline & & Chrysosplenetin \\
\hline & & Casticin \\
\hline & & Quercetagetin-6,7,4'-trimethyl ether \\
\hline & & Quercetagetin-6,7,3',4'-tetramethyl ether \\
\hline & Flavone & Artemetin \\
\hline \multirow{7}{*}{ 2. Terpenoids } & \multirow{5}{*}{ Sesquiterpenoids } & Arteannuic acid \\
\hline & & Arteannuin B \\
\hline & & Artemisinin \\
\hline & & Artemisinic acid \\
\hline & & Deoxy-artemisinin \\
\hline & \multirow{2}{*}{ Triterpenoids (Sterols) } & $\beta$-sitosterol \\
\hline & & Daucosterol \\
\hline \multirow{4}{*}{ 3. Vitamins } & Riboflavin & 7,8-dimethylalloxazine \\
\hline & Niacin & Nicotinic acid \\
\hline & Nucleotide base & Uracil \\
\hline & Aryl ketone & Domesticoside \\
\hline
\end{tabular}

N/B: This classification is based on the reviews by Matsui, et al. [43], Ferreira, et al. [32] \& Brown [17]

Table 4: Docking score of compounds of Artemisia annua plant against antioxidant protein targets $(\mathrm{kcal} / \mathrm{mol})$

\begin{tabular}{rlcccc}
\hline & Compounds & PubChem CID & SOD & CAT & GSH-Px \\
\hline 1 & Trehalose (standard ligand) & 7427 & -5.50 & - & - \\
2 & Metformin (standard ligand) & 4091 & - & -5.40 & - \\
3 & Vitamin E (standard ligand) & 14985 & - & - & -5.80 \\
4 & $3,4-$-di-O-caffeoylquinic acid & 5281780 & -5.90 & -8.40 & -6.50 \\
5 & 3,5 -di-O-caffeoylquinic acid & 6474310 & -7.20 & -7.70 & -7.10 \\
6 & 4,5 -di-O-caffeoylquinic acid & 6474309 & -6.40 & -9.40 & -6.80 \\
7 & Domesticoside & 75072039 & -6.10 & -6.50 & -5.80 \\
8 & Arteannuic acid & 578305 & -5.30 & -6.60 & -6.70 \\
9 & Arteannuin B & 6543478 & -5.80 & -6.90 & -6.50 \\
10 & Artemisinin & 68827 & -6.00 & -7.90 & -6.60 \\
11 & Artemisinic acid & 10922465 & -5.50 & -7.40 & -6.80 \\
12 & Artemetin & 5320351 & -5.90 & -6.80 & -6.60 \\
13 & $\beta$-sitosterol & 222284 & -6.30 & -9.30 & -7.00 \\
14 & $5-O-[(E)-c a f f e o y l] q u i n i c ~ a c i d$ & 25244622 & -6.70 & -7.00 & -6.40 \\
15 & 1,3-di-O-caffeoylquinic acid & 6474640 & -6.40 & -8.70 & -7.20
\end{tabular}


S.A. Ejembi et al. / J. Pharmacy \& Bioresources 18(3), 245-259 (2021)

\begin{tabular}{llcccc}
16 & Chrysosplenol D & 5280699 & -6.10 & -6.80 & -6.90 \\
17 & Chrysosplenetin & 5281608 & -6.20 & -7.60 & -6.80 \\
18 & Casticin & 5315263 & -5.90 & -7.10 & -6.60 \\
19 & Deoxy-artemisinin & 12814879 & -6.00 & -7.00 & -6.40 \\
20 & Daucosterol & 5742590 & -6.50 & -8.60 & -7.00 \\
21 & 7,8 -dimethylalloxazine & 5326566 & -6.50 & -7.60 & -6.70 \\
22 & Methyl-3,4-di-O-caffeoylquinic acid & 5319160 & -7.30 & -7.90 & -6.50 \\
23 & Methyl-3,5-di-O-caffeoylquinic acid & 5319161 & -6.60 & -7.90 & -7.20 \\
24 & Nicotinic acid & 938 & -4.50 & -4.80 & -4.50 \\
25 & p-hydroxybenzoic acid & 135 & -4.50 & -6.50 & -4.70 \\
26 & Quercetagetin-6,7,4'-trimethyl ether & 44259869 & -6.10 & -7.70 & -6.60 \\
27 & Quercetagetin-6,7,3',4'-tetramethyl ether & 14376220 & -6.20 & -6.60 & -6.70 \\
28 & Salicylic acid & 338 & -4.40 & -6.50 & -5.00 \\
29 & Scopoletin & 5280460 & -5.30 & -7.00 & -5.80 \\
30 & Scoparone & 8417 & -5.30 & -6.00 & -5.80 \\
31 & Scopolin & 439514 & -6.20 & -6.80 & -6.40 \\
32 & Uracil & 1174 & -4.40 & -5.30 & -4.00 \\
\cline { 2 - 5 } & & & & &
\end{tabular}

Table 5: Docking score of compounds of Artemisia аппиа plant against oxidative stress protein targets (kcal/mol)

\begin{tabular}{|c|c|c|c|c|c|c|c|}
\hline$\#$ & Compounds & PubChem CID & iNOS & eNOS & Casp-1 & $\mathrm{XOX}$ & NOX \\
\hline 1 & ADMA (standard ligand) & 123831 & -5.80 & -6.40 & - & - & - \\
\hline 2 & Indoprofen (standard ligand) & 3718 & - & - & -6.80 & - & - \\
\hline 3 & 6-mercaptopurine (standard ligand) & 667490 & - & - & - & -4.50 & - \\
\hline 4 & VAS2870 (standard ligand) & 4058452 & - & - & - & - & -7.50 \\
\hline 5 & 3,4-di-O-caffeoylquinic acid & 5281780 & -9.30 & -9.00 & -6.80 & -7.20 & -7.80 \\
\hline 6 & 3,5-di-O-caffeoylquinic acid & 6474310 & -9.10 & -9.40 & -7.40 & -7.40 & -8.30 \\
\hline 7 & 4,5-di-O-caffeoylquinic acid & 6474309 & -8.90 & -9.40 & -6.80 & -7.30 & -7.30 \\
\hline 8 & Domesticoside & 75072039 & -6.90 & -6.90 & -5.80 & -6.70 & -6.40 \\
\hline 9 & Arteannuic acid & 578305 & -7.40 & -7.10 & -5.70 & -6.20 & -6.40 \\
\hline 10 & Arteannuin B & 6543478 & -7.50 & -7.20 & -6.00 & -7.20 & -6.30 \\
\hline 11 & Arte & 68827 & -8.40 & -8.50 & -6.50 & -6.80 & -7.40 \\
\hline 12 & Artem & 10922465 & 6.70 & -6.80 & -5.40 & -6.80 & -6.70 \\
\hline 13 & Arter & 5320351 & -7.50 & -6.80 & -6.00 & -7.00 & -6.30 \\
\hline 14 & $\beta$-sitosterol & 222284 & -8.50 & -6.80 & -6.80 & -7.80 & -6.00 \\
\hline 15 & 5-O-[(E)-caffeoyl]quinic acid & 25244622 & -8.10 & -6.80 & -6.40 & -7.10 & -6.50 \\
\hline 16 & 1,3-di-O-caffeoylquinic acid & 6474640 & -9.60 & -6.80 & -6.90 & -6.70 & -6.20 \\
\hline 17 & Chrysosplenol D & 5280699 & -7.50 & -6.80 & -5.90 & -7.10 & -6.40 \\
\hline 18 & Chrys & 5281608 & -7.60 & -6.80 & -6.10 & -7.10 & -6.50 \\
\hline 19 & & 531 & -7.30 & -6.80 & -5.80 & -7.00 & -6.30 \\
\hline 20 & Deoxy-art & 1281 & -7.80 & -6.80 & -6.20 & -6.80 & -7.30 \\
\hline 21 & Daucosterol & 5742590 & -8.80 & -6.80 & -7.00 & -8.10 & -7.60 \\
\hline 22 & 7,8-dimethylalloxazine & 5326566 & -8.50 & -6.80 & -6.90 & -7.50 & -7.60 \\
\hline 23 & Methyl-3,4-di-O-caffeoylquinic acid & 5319160 & -9.20 & -6.80 & -6.50 & -7.40 & -7.30 \\
\hline 24 & Methyl-3,5-di-O-caffeoylquinic acid & 5319161 & -9.10 & -6.80 & -6.40 & -7.00 & -7.90 \\
\hline 25 & Nicotinic acid & 938 & -5.30 & -6.80 & -4.00 & -4.60 & -4.70 \\
\hline 26 & p-hydroxybenzoic a & 135 & -6.10 & -6.80 & -4.50 & -4.90 & -5.00 \\
\hline 27 & ercetagetin- $6,7,4^{\prime}$-trimethyl & 259869 & -7.90 & -6.80 & -5.90 & -7.30 & -6.50 \\
\hline 28 & Quercetagetin-6,7,3',4'-t & 14376220 & -8.00 & -6.80 & -6.00 & -6.60 & -6.30 \\
\hline 29 & Salicylic acid & 338 & -6.20 & -6.80 & -5.50 & -4.90 & -5.40 \\
\hline 30 & Scop & 5280460 & -6.70 & -6.80 & -5.40 & -5.90 & -5.70 \\
\hline 31 & $\mathrm{Sc}$ & 8417 & -7.00 & -6.80 & -5.20 & -6.00 & -5.60 \\
\hline 32 & Scopolin & 439514 & -8.20 & -6.80 & -6.10 & -7.50 & -6.70 \\
\hline 33 & Uracil & 1174 & -5.10 & -6.80 & -4.10 & -4.30 & -4.70 \\
\hline
\end{tabular}


Table 6: Active site directed docking score of compounds of Artemisia annua plant against antioxidant protein

\begin{tabular}{|c|c|c|c|c|c|}
\hline \multicolumn{6}{|c|}{ targets $(\mathrm{kcal} / \mathrm{mol})$} \\
\hline \# & Compounds & PubChem CID & SOD & CAT & GSH-Px \\
\hline 1 & Trehalose (standard ligand) & 7427 & 43.30 & - & - \\
\hline 2 & Metformin (standard ligand) & 4091 & - & 4.80 & - \\
\hline 3 & Vitamin E (standard ligand) & 14985 & - & - & - \\
\hline 4 & 3,4-di-O-caffeoylquinic acid & 5281780 & 90.60 & 76.70 & - \\
\hline 5 & 3,5-di-O-caffeoylquinic acid & 6474310 & 92.50 & 94.90 & - \\
\hline 6 & 4,5-di-O-caffeoylquinic acid & 6474309 & 93.60 & 92.00 & - \\
\hline 7 & Domesticoside & 75072039 & 56.60 & 31.80 & - \\
\hline 8 & Arteannuic acid & 578305 & 50.80 & 19.80 & - \\
\hline 9 & Arteannuin B & 6543478 & 51.90 & 33.70 & - \\
\hline 10 & Artemisinin & 68827 & 75.00 & 58.10 & - \\
\hline 11 & Artemisinic acid & 10922465 & 42.20 & 21.60 & - \\
\hline 12 & Artemetin & 5320351 & 88.70 & 54.70 & - \\
\hline 13 & $\beta$-sitosterol & 222284 & 122.30 & 85.60 & - \\
\hline 14 & 5-O-[(E)-caffeoyl]quinic acid & 25244622 & 55.60 & 38.60 & - \\
\hline 15 & 1,3-di-O-caffeoylquinic acid & 6474640 & 96.10 & 86.70 & - \\
\hline 16 & Chrysosplenol D & 5280699 & 89.50 & 52.80 & - \\
\hline 17 & Chrysosplenetin & 5281608 & 87.40 & 51.70 & - \\
\hline 18 & Casticin & 5315263 & 85.10 & 63.60 & - \\
\hline 19 & Deoxy-artemisinin & 12814879 & 64.40 & 39.90 & - \\
\hline 20 & Daucosterol & 5742590 & 154.60 & 143.40 & - \\
\hline 21 & 7,8-dimethylalloxazine & 5326566 & 56.30 & 31.80 & - \\
\hline 22 & Methyl-3,4-di-O-caffeoylquinic acid & 5319160 & 94.90 & 88.40 & - \\
\hline 23 & Methyl-3,5-di-O-caffeoylquinic acid & 5319161 & 97.10 & 101.10 & - \\
\hline 24 & Nicotinic acid & 938 & 3.90 & 0.80 & 49.10 \\
\hline 25 & p-hydroxybenzoic acid & 135 & 6.80 & 0.00 & 83.50 \\
\hline 26 & Quercetagetin-6,7,4'-trimethyl ether & 44259869 & 83.20 & 45.50 & - \\
\hline 27 & Quercetagetin-6,7,3',4'-tetramethyl ether & 14376220 & 83.70 & 53.80 & - \\
\hline 28 & Salicylic acid & 338 & 5.90 & 0.20 & 61.60 \\
\hline 29 & Scopoletin & 5280460 & 20.60 & 7.00 & - \\
\hline 30 & Scoparone & 8417 & 25.90 & 14.60 & - \\
\hline 31 & Scopolin & 439514 & 74.60 & 42.70 & - \\
\hline 32 & Uracil & 1174 & 1.00 & -2.00 & 29.10 \\
\hline
\end{tabular}

Table 7: Active site directed docking score of compounds of Artemisia annua plant against xanthine oxidase and caspase-1 ( kcal/mol)

\begin{tabular}{ccccc}
\hline$\#$ & Compounds & PubChem CID & XOX & Casp-1 \\
\hline 1 & 6-mecaptopurine (standard ligand) & 667490 & -4.6 & - \\
2 & Allopurinol (standard ligand) & 135401907 & -5.7 & - \\
3 & Acyclovir (standard ligand) & 1353985213 & -6.4 & - \\
4 & Fenbufen (standard ligand) & 3335 & - & -6.3 \\
5 & Indoprofen (standard ligand) & 3718 & - & -6.8 \\
6 & Aspirin (standard ligand) & 2244 & - & -5.0 \\
7 & 3,4-di-O-caffeoylquinic acid & 5281780 & 3.10 & 99.00 \\
8 & 3,5-di-O-caffeoylquinic acid & 6474310 & 4.70 & 68.80 \\
9 & 4,5-di-O-caffeoylquinic acid & 6474309 & 2.50 & 79.60 \\
10 & Domesticoside & 75072039 & -2.60 & 12.70 \\
11 & Arteannuic acid & 578305 & -5.30 & 10.80 \\
12 & Arteannuin B & 6543478 & -5.40 & 40.50 \\
13 & Artemisinin & 68827 & -0.80 & 33.80 \\
14 & Artemisinic acid & 10922465 & -5.30 & 17.60 \\
15 & Artemetin & 5320351 & -5.50 & 98.90 \\
16 & $\beta-$ sitosterol & 222284 & 0.70 & 80.80 \\
17 & 5-O-[(E)-caffeoyl]quinic acid & 25244622 & -6.10 & 25.60
\end{tabular}


S.A. Ejembi et al. / J. Pharmacy \& Bioresources 18(3), 245-259 (2021)

\begin{tabular}{lcccc}
8 & 1,3-di-O-caffeoylquinic acid & 6474640 & 0.20 & 91.90 \\
19 & Chrysosplenol D & 5280699 & -5.60 & 35.00 \\
20 & Chrysosplenetin & 5281608 & -5.50 & 78.00 \\
2 & Casticin & 5315263 & -5.60 & -5.80 \\
23 & Deoxy-artemisinin & 12814879 & -2.70 & -6.20 \\
4 & Daucosterol & 5742590 & 5.70 & -7.00 \\
5 & 7,8-dimethylalloxazine & 5326566 & -8.10 & -6.90 \\
26 & Methyl-3,4-di-O-caffeoylquinic acid & 5319160 & 0.90 & -6.50 \\
7 & Methyl-3,5-di-O-caffeoylquinic acid & 5319161 & 2.50 & -6.40 \\
28 & Nicotinic acid & 938 & -5.90 & -4.00 \\
29 & Quercetagetin-6,7,4'-trimethyl ether & 135 & -6.70 & -4.50 \\
30 & Quercetagetin-6,7,3',4'-tetramethyl ether & 14259869 & -6.70 & -5.90 \\
1 & Salicylic acid & 338 & -4.50 & -6.00 \\
32 & Scopoletin & 5280460 & -6.70 & -5.50 \\
33 & Scoparone & 8417 & -6.10 & -5.40 \\
34 & Scopolin & 439514 & -4.50 & -6.10 \\
35 & Uracil & 1174 & -5.10 & -4.10 \\
\hline
\end{tabular}

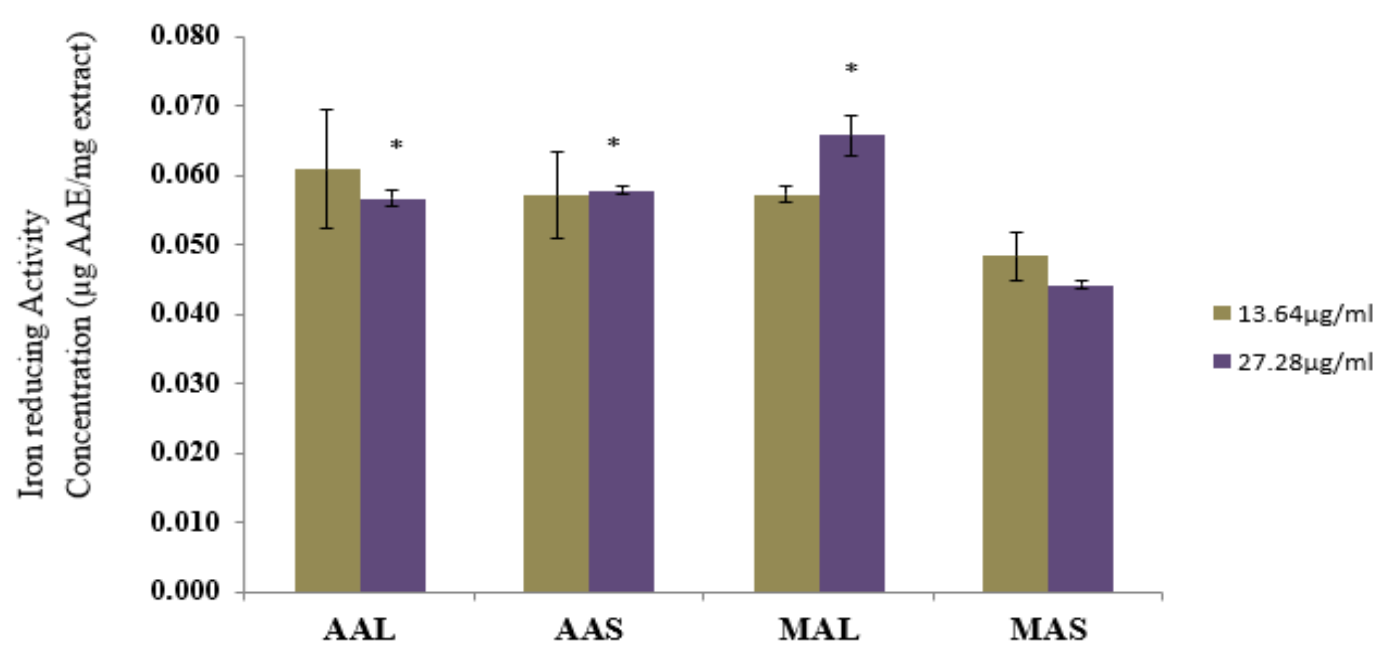

Figure 1: Iron reducing power of aqueous and methanol extracts of Artemisia annиa leaf and seed Values are mean \pm SEM of triplicate results $(n=3)$. (*) indicates group that is statistically significant as compared to MAS at 27.28 $\mu \mathrm{g} / \mathrm{ml}$. Differences are considered significant at $p<0.05$ with Two-way ANOVA and the Bonferioni post-hoc t-test correction factor. (AAE-Ascorbic acid Equivalent)

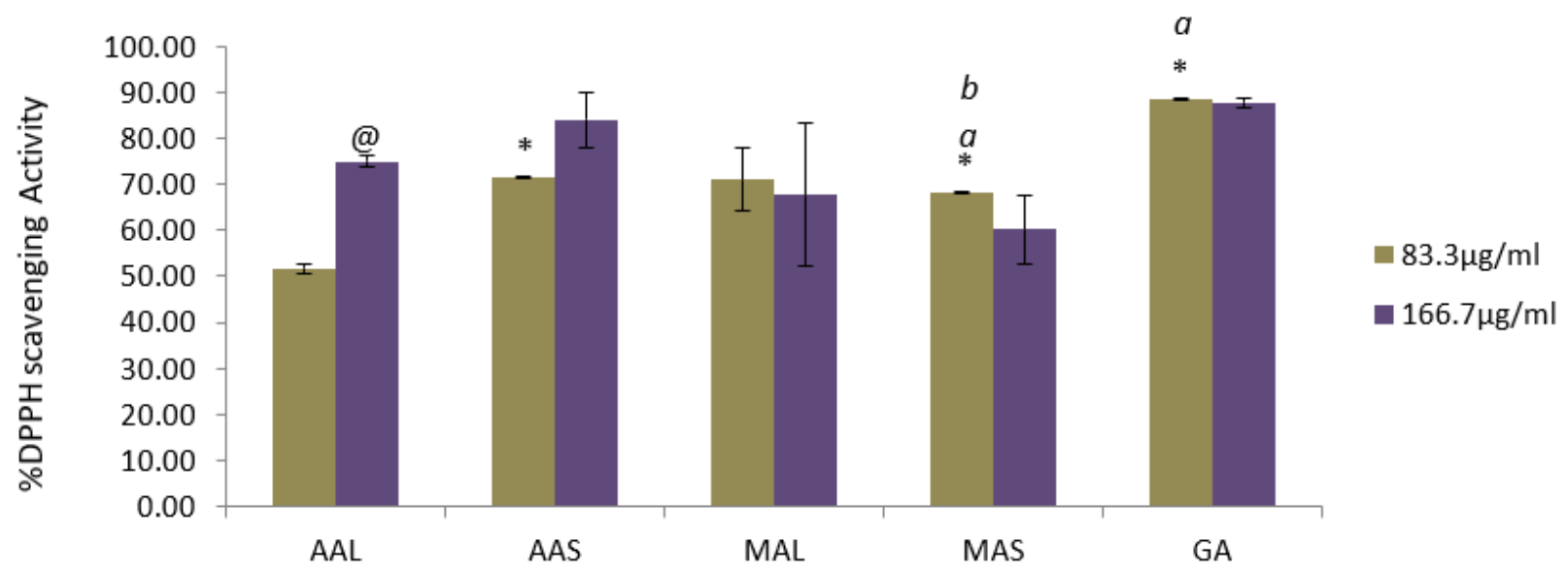

Figure 2: DPPH scavenging activities of aqueous and methanol extracts of Artemisia annua leaf and seed 
Values are mean \pm SEM of triplicate results $(n=3)$. $\left({ }^{@}\right)$ indicates difference in the DPPH scavenging activity between $83.3 \mu \mathrm{g} / \mathrm{ml}$ and $166.7 \mu \mathrm{g} / \mathrm{ml}$. (*) indicates group that is statistically significant as compared to AAL at $83.3 \mu \mathrm{g} / \mathrm{ml}$ and the superscripts $\left(^{(a, b}\right)$ indicate groups that are statistically significant as compared to AAS and MAS respectively at $83.3 \mu \mathrm{g} / \mathrm{ml}$. Differences are considered significant at $p<0.05$ with Two-way ANOVA with the

Bonferioni post-hoc $t$ - test correction factor
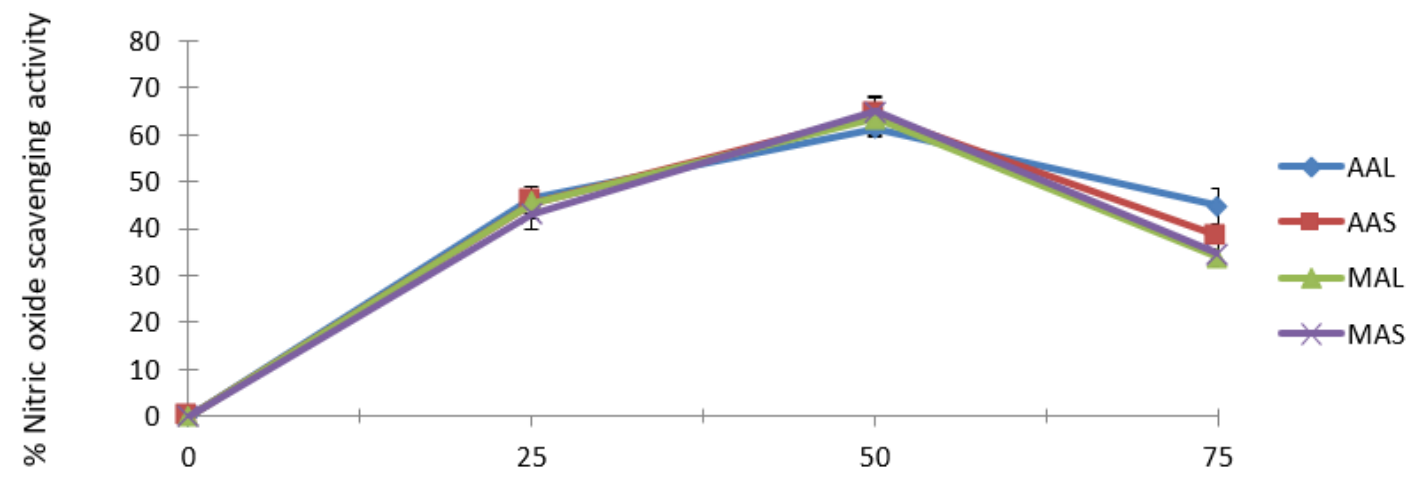

Figure 3: Nitric oxide scavenging activities of aqueous and methanol extracts of Artemisia annиa leaf and seed There is no significant difference in the nitric oxide radical scavenging ability of extracts of the different solvent system used.

Molecular docking interactions (site directed docking). The active sites of targets were accessed as deposited in the Computed Atlas of Surface Topography of protein (CASTp) database [40] to assess the binding interactions of ligands and the amino acid side chains positioned at the active site of these proteins. The results of our study revealed that the bioactive compounds of $A$. апnи a and the standard ligands had a positive change in Gibb's energy $(\Delta \mathrm{G})(\mathrm{kcal} / \mathrm{mol})$ in the docking interaction with the antioxidant target proteins, except for uracil $(-2.00 \mathrm{kcal} / \mathrm{mol})$ in the docking against $C A T$ (Table 6). Following the fact that the more negative the binding energy, the better the protein-ligand association and the stability of complex [41], our high docking scores $(-\Delta \mathrm{G} \mathrm{kcal} / \mathrm{mol})$ in the non-site specific docking against these antioxidant proteins (Table 5) suggest that these ligands are not inhibitors of SOD, CAT and GSH-PX.

Protein active site directed docking against oxidative stress targets (Casp- 1 and $X O X)$ revealed that 7,8-dimethylalloxazine ($8.10 \mathrm{kcal} / \mathrm{mol})$, Scopoletin $(-7.90 \mathrm{kca} / \mathrm{mol})$ were among the five (5) topmost compounds in their binding affinity to the active site of $X O X$ than acyclovir $(-6.40 \mathrm{kcal} / \mathrm{mol})$, a known xanthine oxidase inhibitor. Similarly, 7,8dimethylalloxazine $(-6.90 \mathrm{kcal} / \mathrm{mol})$ and Daocosterol (-7.00 $\mathrm{kcal} / \mathrm{mol})$ had higher docking scores than indoprofen (-6.80 $\mathrm{kcal} / \mathrm{mol}$ ) against casp-1 (Table 7). The molecular (3D and 2D) interactions of indoprofen $(-6.80 \mathrm{kcal} / \mathrm{mol})$, daucosteroal ($7.00 \mathrm{kcal} / \mathrm{mol}$ ) and 7, 8-dimethylalloxazine ($6.90 \mathrm{kcal} / \mathrm{mol}$ ) against Casp-1 are expressed in Figure 4, and those of acyclovir (-6.40 $\mathrm{kcal} / \mathrm{mol}), \quad 7, \quad 8$-dimethylalloxazine $(-8.10$ $\mathrm{kcal} / \mathrm{mol}$ ) and scopoletin (-7.90) against XOX are shown in Figure 5.

Conventional $\mathrm{H}^{+}$bond, Van der waals' force, Pi-akyl bond, Pi-carbon and Carbon hydrogen bonds were among the several bond interactions involved in the ligand-target binding. The conventional $\mathrm{H}^{+}$bond is said to be stable and a reliable bond in this interaction [42]. In Figure 4, indoprofen (A), daucosterol (B), and 7, 8-dimethylalloxazine (C) interacted with conventional $\mathrm{H}^{+}$bonds to at least two amino acids at the active site of Casp-1 (ARG 161, ARG 163 and TYR 198) with several other amino acids in which they share other bond types. The interaction with xanthine oxidase (Figure 5) reveals that acyclovir (D), 7, 8-dimethylalloxazine (E) and scopoletin (F), 
bond with at least five conventional $\mathrm{H}^{+}$bonds with the amino acids, GLU 802, ALA 1079, THR 1010, VAL 1011 and ARG 880.
Acyclovir, however, had a weaker binding (van der waals') force with ALA 1079 and ARG 880 .

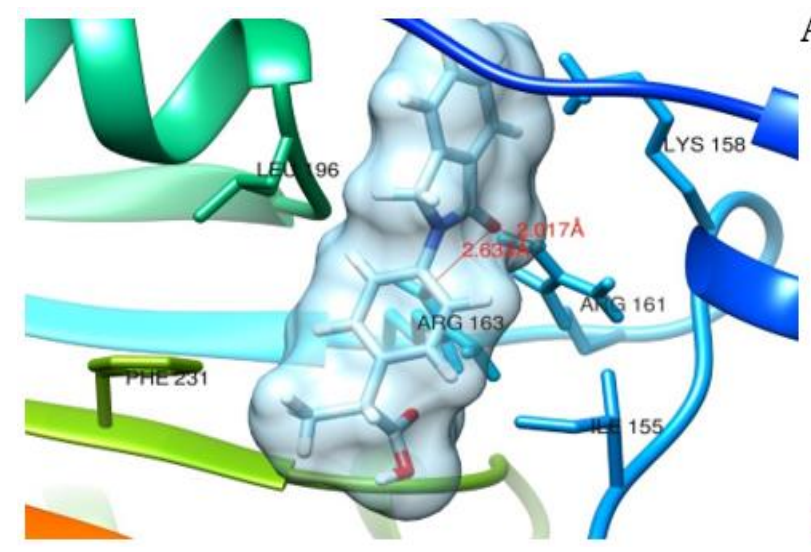

A

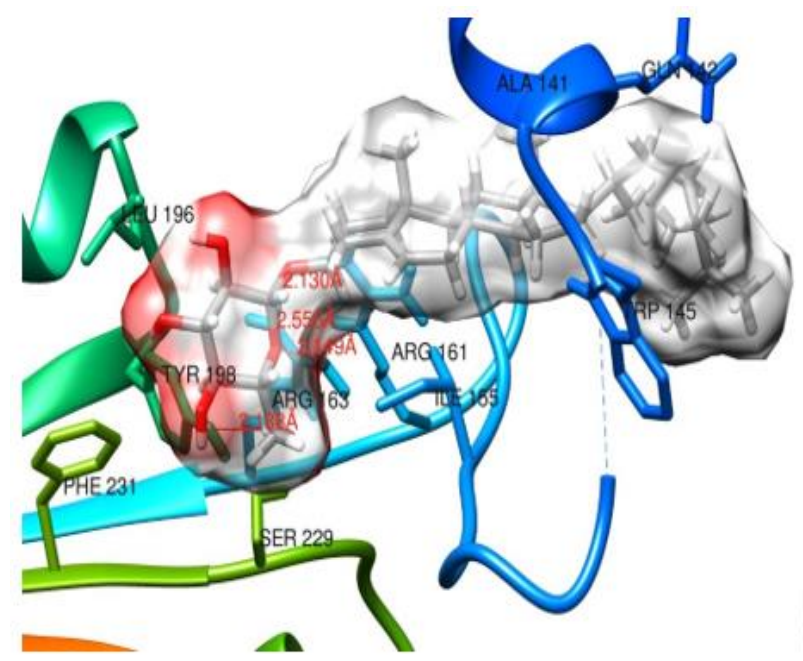

B



$\mathrm{C}$
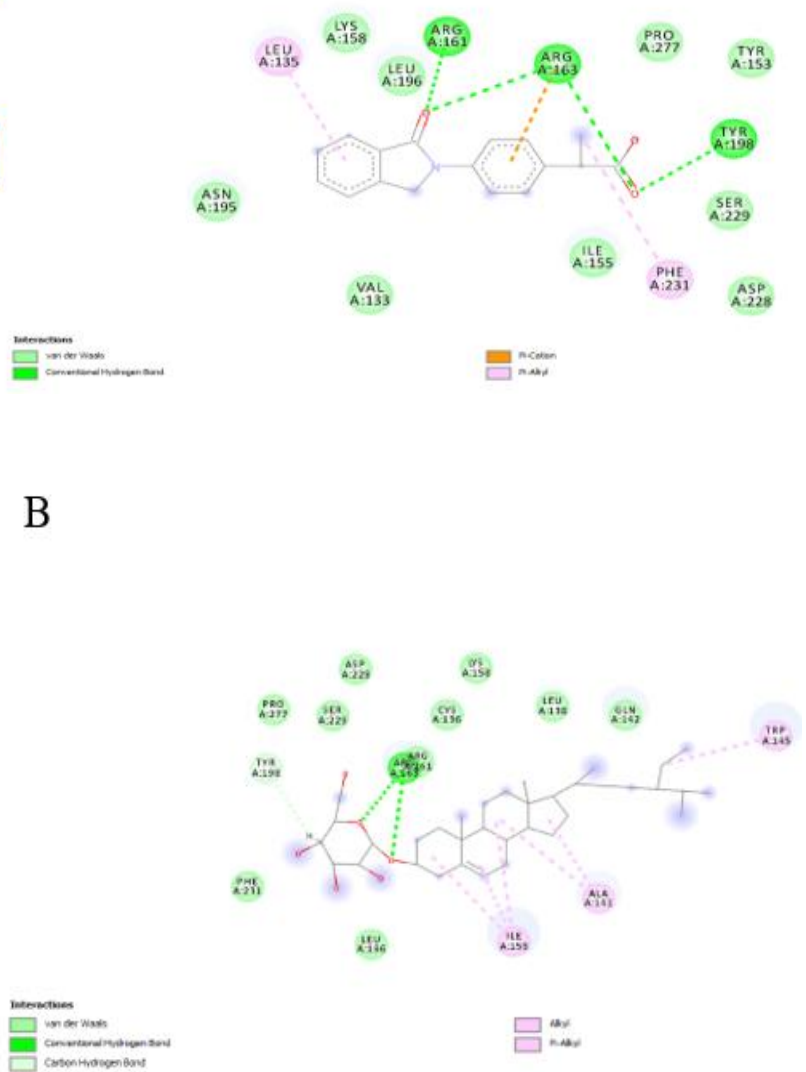

큼서

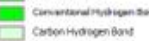

吕总

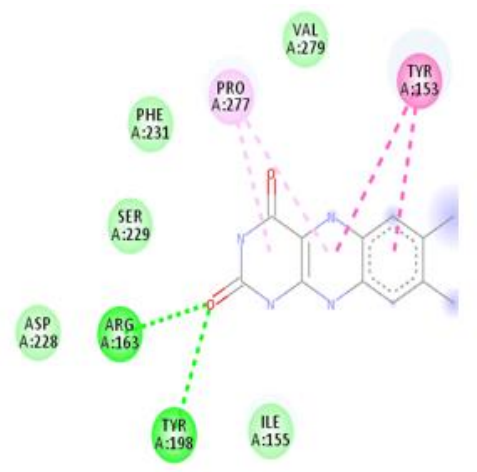

Figure 4: 3D (left) and 2D (right) interactions of indoprofen (A), daucosterol (B) and 7, 8 - dimethylalloxazine (C) with caspase- 1 

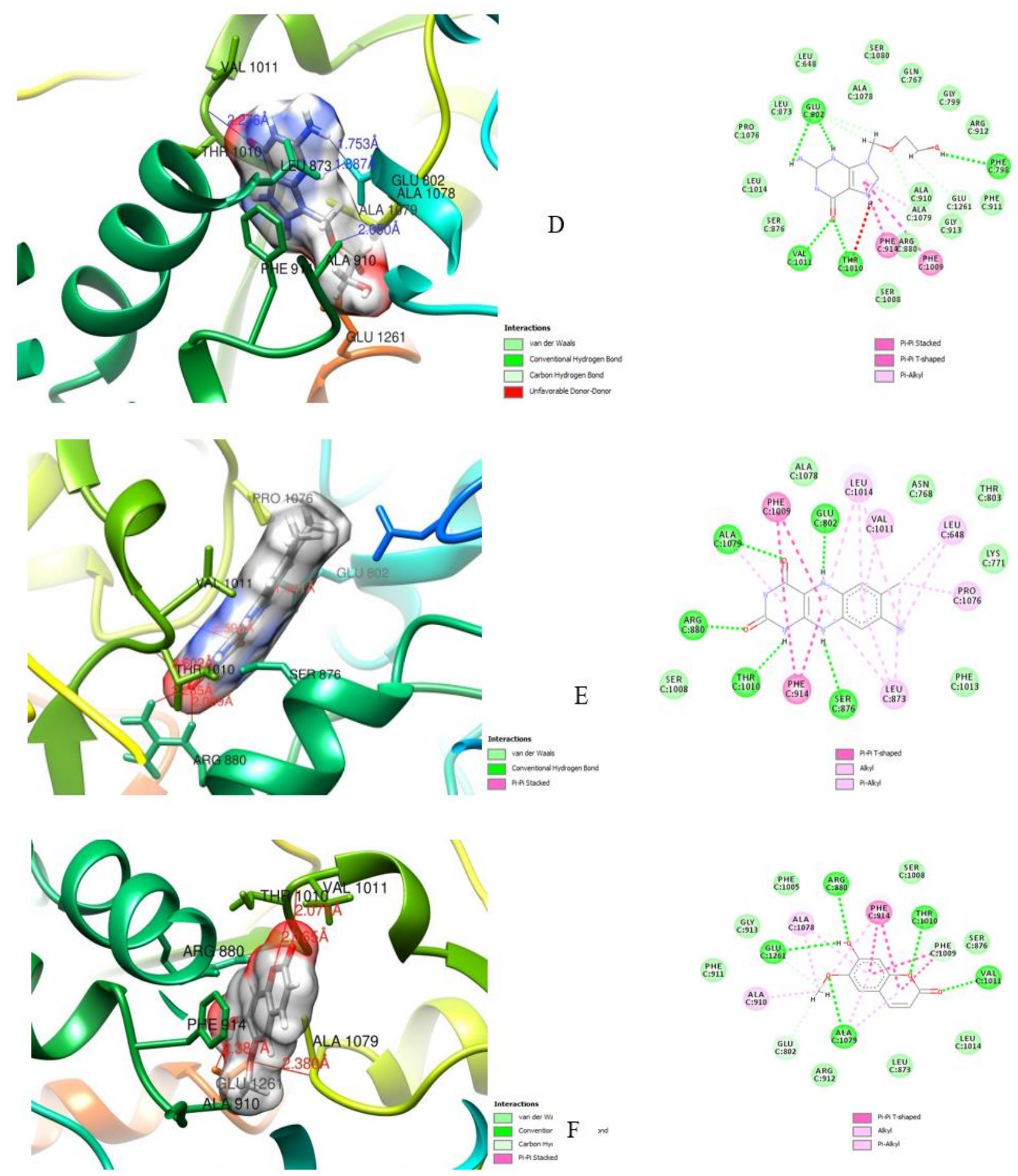

Figure 5: 3D (left) and 2D (right) interactions of acyclovir (D), 7, 8 - dimethylalloxazine (E) and scopuletin (F) with xanthine oxidase

These interactions (Figure 4 \& 5) suggest therefore a stable binding of these bioactive compounds of $A$. аппиа to these targets, and daucosterol, 7,8- dimethylalloxazine, scopoletin may be good substitutes for the existing target inhibitors that are known with adverse effects. 
Conclusion. Plant extracts of Artemisia annua leaf and seed from Gangnim showed reasonable antioxidant activity. Literature reports that antioxidants reduce oxidative stress in cells and are therefore useful in the treatment of many human diseases, including cancer. The antioxidant assay, molecular docking scores and molecular interactions of compounds of $A$. annua with respective protein targets used is suggestive of $A$. annua plant having the prospect to prevent oxidative stress and cure pathological conditions that may arise due to oxidative stress.

Acknowledgment. We are grateful to Prof. John C. Aguyi of Africa Center of Excellence in Phytomedicine Research and Development (ACEPRD), University of Jos, Nigeria for generously donating the Artemisia annua leaf and seed used for this study, and $\mathrm{Mr}$. Adegboyega E. Abayomi, a postgraduate student of the Department of Biochemistry, Faculty of Basic Medical Sciences, University of Jos, for lending his computational biology skill for this study.

\section{REFERENCES}

1. Emmanuel AO, Yewubdar O, Jonah YP, Modupe IB, Aguiyi JC. Phytochemical Screening and In vivo Antiplasmodial Sensitivity Study of Locally Cultivated Artemisia annua Leaf Extract against Plasmodium berghei. American Journal of Ethnomedicine, 2014 1(1): 042 - 049

2. Khanal P. Antimalarial and anticancer properties of artesunate and other artemisinins: current development. Monatsh Chem 2021 152:387-400 https://doi.org/10.1007/s00706-021-02759-X

3. Mesa LE, Lutgen P, Velez ID, Segura AM, Robledo SM. Artemisia annua L., Potential Source of Molecules with Pharmacological Activity in Human Diseases. AJPCT. 2015 3(05): 436 - 450.

4. Nkuitchou-Chougouo RDK, Fomekong DF, Kouamouo J, et al. Physicochemical Characteristics of Artemisia annua, an Antimalarial Plant from the Grass- field Regions of Cameroon. International Journal of Engineering Science and Computing, 2016 6(3): $\quad 2762 \quad-\quad 2769$. http://hdl.handle.net/123456789/2333
5. Nigam M, Atanassova M, Mishra AP, et al. Bioactive Compounds and Health Benefits of Artemisia Species. Natural Product Communications, 20191 17. sagepub. com/ journals- permissions DOI: 10.1177/1934578X19850354 journals. sagepub. com/ home/ npx

6. Inagi R. Oxidative stress in cardiovascular disease: A new avenue toward future therapeutic approaches. Frontiers in Cardiovascular Drug Discovery, 2010 1: $138-153$.

7. Mandal A. What is Oxidative Stress? New-Medical $2021<$ https://www.news-medical.net/health/Whatis-Oxidative-Stress.aspx $>$.

8. Pihlanto A, Akkanen S, Korhonen HJ. ACEinhibitory and antioxidant properties of potato (Solanum tuberosum). Food Chem., 2008 109: 104 112.

9. Eldridge L. Free Radicals: Definition, Causes, Antioxidants, and Cancer. What Exactly Are Free Radicals and Why Are They Important? 2020. https://www.verywellhealth.com/information-aboutfree-radicals-2249103

10. Lai F, Wen Q, Li L, Wu H, Li X. Antioxidant activities of water-soluble polysaccharide extracted from mung bean (Vigna radiate L.) hull with ultrasonic assisted treatment. Carbohydr. Polym. 2010 81: 323 - 329.

11. Czerska M, Mikołajewska K, Zieliński M, Gromadzińska J, Wąsowicz W. Today's oxidative stress markers. Med Pr. 2015;66(3):393-405. doi: 10.13075/mp.5893.00137. PMID: 26325052.

12. Oberley JO, Oberley L. Antioxidant enzyme levels in cancer. Histopathol, 1997 12: 525-535.

13. Anagnostopoulou MA, Kefalas P, Papageorgiou VP, Assimopoulou AN, Boskou D. Radical scavenging activity of various extracts and fractions of sweet orange peel (Citrus sinensis). Food Chem., 2006 94: $19-25$.

14. Yingming $\mathrm{P}$, Ying L, Hengshan W, Min L. Antioxidant activities of several Chinese medicine herbs. Food Chem., 2004 88: 347 - 350.

15. Shahid I, Umer Y, Kim WC, Muhammad ZMI. Chemical Composition of Artemisia annua L. Leaves and Antioxidant Potential of Extracts as a Function of Extraction Solvents. Molecules, 2012 17: 6020 -6032 .

16. Kivits GAA, Vamdersman FJP, Tijburg LBM. Analysis of Catechin from Green and Black tea in Humans: a specific and sensitive colorimetric assay of total catechins in biological fluids. Int. J food Sci. Nutr., 1997 48: 387 - 392. 
17. Brown GD. The biosynthesis of artemisinin (Qinghaosu) and the phytochemistry of Artemisia annua L. (Qinghao). Molecules. 2010 Oct 28;15(11):7603-98.

doi: 10.3390/molecules15117603. PMID: 21030913; PMCID: PMC6259225.

18. Asuzu, 1986 In Emmanuel AO, Yewubdar O, Jonah YP, Modupe IB, Aguiyi JC. Phytochemical Screening and In vivo Antiplasmodial Sensitivity Study of Locally Cultivated Artemisia annua Leaf Extract against Plasmodium berghei. American Journal of Ethnomedicine. 2014 1(1): 042 - 049

19. Chu Y, Sun J, Wu X, Liu R. Antioxidant and antiproliferative activities of common vegetables. Journal of Agricultural and Food Chemistry, 2002 49(7): $3216-3222$.

20. Harborne JB. Phytochemical methods. A Guide to Modern Techniques in Plants Analysis. $19842^{\text {nd }}$ Edition. 1 - 10, 100 - 117

21. Sofowora A. Medical Plants and Traditional Medicine in Africa. 1993289 pages

22. Trease, GE, Evans, WC. Pharmacognosy. 13th Edition, Baillière Tindall, London (1989)

23. Singleton VL, Orthofor R, Lamuela-Raventos RM. Analysis of total phenols and other oxidation substrates and antioxidants by means of FolinCiocalteau reagent. Method. In Enzymology, 1999 299:152-178. https://doi.org/10.1016/S00766879(99)99017-1

24. Meda A, Lamien CE, Romito M, Millogo J, Nacoulma OG. Determination of the total phenolic, flavonoid and prolinecontents in Burkina Faso honey, as well as their radical scavenging activity. Food Chem, 2005 91:571-577

25. Oyaizu M. Studies on products of browning reaction: antioxidative activity of products of browning reaction prepared from glucosamine. Japan Journal of Nutrition, 1986 44: 307 - 315. doi: 10.5264/eiyogakuzashi.44.307.

26. Gyamfi MA, Yonamine M, Aniya Y. Free-radical scav-enging action of medicinal herbs from Ghana: Thonningia san-guinea on experimentally-induced liver injuries. General Pharmacology, 1999 32: 661 667. doi: 10.1016/S0306-3623(98)00238-9.

27. Marcocci L, Maguire JJ, Droy-Lefaix MT, Packer L. The nitric oxide-scavenging properties of Ginkgo biloba extract EGb 761. Biochem Biophys Res Commun. 1994 Jun 15;201(2):748-55. doi: 10.1006/bbrc.1994.1764. PMID: 8003011.

28. www.wwpdb.org Berman, HM, Henrick, K, Nakamura, H. Announcing the worldwide Protein
Data Bank Nature Structural Biology 200310 (12): 980.

29. Singh P, Kumar R, Tiwari S, Khanna RS, Tewari AK, Khanna HD. Docking, Synthesis and Evaluation of Antioxidant Activity of 2,4,5-Triaryl Imidazole. Clin Med Biochem, 2015 Open Access 1(1):1 - 4. DOI: $10.4172 / 2471-2663.1000105$

30. Rana S, Dixit S, Mittal A. In Silico Target Identification and Validation for Antioxidant and Anti-inflammatory Activity of Selective Phytochemicals. Brazilian Archives of Biology and Technology, 2019 62(4): 1 - 11. DOI:10.1590/16784324-2019190048

31. Johnson TO, Odoh KD, Nwonuma CO, Akinsanmi AO, Adegboyega AE. Biochemical evaluation and molecular docking assessment of the antiinflammatory potential of Phyllanthus nivosus leaf against ulcerative colitis. Heliyon. 2020 6(5):e03893. doi: 10.1016/j.heliyon.2020.e03893.

32. Ferreira JF, Luthria DL, Sasaki T, Heyerick A. Flavonoids from Artemisia annua L. as antioxidants and their potential synergism with artemisinin against malaria and cancer. Molecules. 2010 Apr 29;15(5):3135-70. doi: 10.3390/molecules15053135. PMID: 20657468; PMCID: PMC6263261.

33. Akinsanmi AO, Johnson OT, Longdet IY. In Vitro Evaluation of Free Radical Scavenging, Fe2+ and SNP-Induced Lipid Peroxidation (Rat Brain) Activities of Methanolic Extracts from Three (3) Northern Nigerian Plants Leaf. Journal of Tropical Life $\quad$ Science. $\quad 2019 \quad$ 9(1). http://dx.doi.org/10.11594/jtls.09.01.xx

34. Mohammad MH. Study the Effect of Cold and Hot Water Extracts of Parsley Plant Petroselinumcrispum on the Growth of some Enterobacteriaceae. Journal of Al-Nahrain University, 2014 17(1): 148 - 154.

35. Oboh G, Ademosun AO. Characterization of the antioxidant properties of phenolic extracts from some citrus peels. J Food Sci Technol, 2012 49(6): 729 736. doi:10.1007/s13197-010-0222-y

36. Imam MU, Zhang S, Ma J, Wang H, Wang F. Antioxidants Mediate Both Iron Homeostasis and Oxidative Stress. Nutrients. 2017 Jun 28;9(7):671. doi: 10.3390/nu9070671. PMID: 28657578; PMCID: PMC5537786.

37. Tae K, Kim S. Inhibition of iNOS and DNA Oxidation by Methanol Extract of Schizonepeta tenuifolia Trop J Pharm Res, June 201211 (3): 397 $404 \quad$ http://www.tjpr.org http://dx.doi.org/10.4314/tjpr.v11i3.8

38. Ma X, Deng D, Chen W. Inhibitors and Activators of SOD, GSH-Px, and CAT. Intech open science, 
$2017207 \quad-\quad 224 . \quad$ DOI: 10.5772/65936. https://www.intechopen.com/chapters/52877

39. Wong VW, Lerner E. Nitric oxide inhibition strategies. Future Sci OA. 2015;1(1):FSO35. doi: 10.4155/fso.15.35. PMID: 26634146; PMCID: PMC4664049.

40. sts.bioe.uic.edu

41. Du X, Li Y, Xia YL, Ai SM, Liang J, Sang P, Ji XL, Liu SQ. Insights into Protein-Ligand Interactions: Mechanisms, Models, and Methods. Int J Mol Sci. 2016 Jan 26;17(2):144. doi: 10.3390/ijms17020144. PMID: 26821017; PMCID: PMC4783878.

42. Chen D, Oezguen N, Urvil P, Ferguson C, Dann SM, Savidge TC. Regulation of protein-ligand binding affinity by hydrogen bond pairing. Sci Adv. 2016 Mar 25;2(3):e1501240. doi: 10.1126/sciadv.1501240. PMID: 27051863; PMCID: PMC4820369.

43. Matsui Y, Nakamura S, Kondou N, Takasu Y., Ochiai R., Masukawa Y. Liquid chromatographyelectrospray ionization-tandem mass spectrometry for simultaneous analysis of chlorogenic acids and their metabolites in human plasma. Journal of chromatography. B, Analytical Technologies in the Biomedical and Life Sciences. 2007 Oct; 858(12):96-105. DOI: 10.1016/j.jchromb.2007.08.013. PMID: 17766198. 